\title{
A Semiotic Approach to the Design of Agent- mediated E-commerce Systems
}

\author{
Samuel Chong and Kecheng Liu \\ School of Computing, Staffordshire University, Beaconside, Stafford ST18 ODG
}

Key words: E-commerce, Agents, Agent communication language, Organisational semiotics, Semantic analysis, Norm analysis

Abstract: The emergence of the Internet and electronic commerce (or e-commerce) has opened up a new dimension for conducting business. Incorporating agent technology into e-commerce systems has brought even more potential to the improvement of efficiency and effectiveness of business systems. While the work and technology of agent-mediated e-commerce starts to benefit the industry and economy, much research in the theory, methods and technologies is needed to achieve the return of value of e-commerce in a larger scale. In agent-mediated e-commerce systems, issues such as the delegation of authorities from a human user to a machine agent need to be dealt with. Also, for agents to communicate with one another, there is a need for techniques for specifying a common vocabulary of terms to be used.

This paper presents a semiotic approach to evaluating and addressing these problems. It presents a set of methods for the design of agent-mediated ecommerce systems. A case study of an online bookstore is described to demonstrate how the design of an agent-mediated e-commerce system can be conducted using the semiotic approach. The approach offers a way of identifying the information that should be shared by agents. It also offers a method to represent and handle propositional attitudes and make explicit link between the attitudes and intentions, beliefs, perceptions, acts and values.

\section{INTRODUCTION}

Electronic commerce (or e-commerce) is already considered to be a multi-billion dollar industry, as the emergence of e-commerce has brought in

The original version of this chapter was revised: The copyright line was incorrect. This has been corrected. The Erratum to this chapter is available at DOI: 10.1007/978-0-387-35500-9_30 
a new and effective means of conducting business and reaching potential customers.

New paradigms and theories of business have provided frameworks for conceiving and designing e-commerce systems, such as virtual organisation (Mowshowitz, 1997) and distributed enterprise (Liu et al., 1999). Emerging technologies such as software agents have enabled the implementation of these systems, especially the agent mediated e-commerce systems. These systems have their special features because they have direct impact on the business processes and practices within an organisation, and they are open systems interacting with a broad range of users. Methods and techniques for e-commerce systems development therefore are needed to cope with these features.

The uniqueness of agent-mediated e-commerce makes it complex to use existing requirements analysis methods. For example, an agent has roles and intentions and we need a formal method that is able to specify that. Our research adopts the semiotic framework and semiotic methods for requirements analysis and systems design for agent-mediated e-commerce.

In section 2, agents and e-commerce and the problems of agent-mediated e-commerce will be discussed. Section 3 and 4 discuss the theoretical foundations such as organisational semiotics and speech act theory. The former is to be adopted for critical analysis of agent communication; while the latter serves as a conceptual ground for current work and future development of agent communication languages. In section 4 , we evaluate a widely used agent communication language, KQML, using the semiotic framework. We also look at the inadequacy of KQML to be used in an agent-mediated electronic commerce setting. Section 5 presents the semiotic methods for the design of an agent-mediated e-commerce system with an illustration of a case study. This section also highlights the possibility of identifying the internal mental states of agents such as beliefs and intentions using norm analysis. Section 6 summaries the work and indicates some directions for further work.

\section{AGENTS AND E-COMMERCE}

In this section, an introduction of e-commerce and agents will be given. The main objective is to identify the problems in designing an agentmediated e-commerce system. 


\subsection{Electronic Commerce}

E-commerce has been defined as the ability to share a wide range of communiqué and data with trading partners (Adams, 1994). The components that make up e-commerce could be the Internet, the e-mail networks such as MCI Mail, Compuserve, groupware systems such as LotusNotes, Wordperfect Office and many others (Adams, 1994). Some of the technologies that make up e-commerce are Electronic Data Interchange (EDI), bar coding, fax, imaging and smart cards (Spence, 1994). Business data sent in e-commerce consist of both structured and unstructured data such as electronic memos send via e-mail (Fisher, 1996).

E-commerce is emerging as an important avenue for companies to reach their prospective buyers in the world of business today. One reason for its attractiveness is its low cost (Lederer, et al., 1996). It is having and will continue to have a dramatic impact on business into the $21^{\text {st }}$ century (Hornback, 1995). E-commerce can be seen as an important means for companies to implement business strategies. In this aspect, productivity, cash flow can be increased, inventory can be decreased and relationships with customers can be enhanced (Boynton, 1994).

The fast emerging technologies have widened the scopes of electronic commerce. When the scopes expand, there is also an increased need for agents to support the processes. Agents-mediated electronic commerce needs to interpret a wider range of meanings. Kimbrough \& Moore (1997) called this 'the requirement for expressive felicity'. This means that the semantic meanings in the interaction between different parties or agents in an electronic commerce setting needs to be sent and encoded in an unambiguous way. The next section will discuss the definition of agency and their workings.

\subsection{Definition of Agency and Agents}

One of the most influential researches in Agency theory is due to the work of Cohen and Levesque (1990a, 1990b). Their contributions have been used to develop a theory of intention (as in 'I intent to..'), which forms the pre-requisite for applying the theory of speech acts (Searle, 1969).

The fundamental aspect of agency theories is the delegation of duties and responsibilities to a person or machine whom we call an Agent. It is almost impossible to know where to look for things when we need it, especially information that is available across global computer networks. Therefore agents are created to help us find information that we need as quickly as possible. 
Over the past years, there has been a lot of interest in agent systems (Shoham, 1993). There is an increased need for agents to support both customers and suppliers in electronic commerce. "Agents are important because they let software components interoperate within modern applications like electronic commerce" (Singh, 1998). However, it is not easy to reach a common consensus on the definition of agents. As with object technology a decade ago, it is difficult for anyone to agree even on the basic question of what an agent is (Nwana and Wooldridge, 1996). According to Finin et al. (1997), anything might qualify as an agent these days. Several definitions coexist and each definition has different emphasis on the properties of agents. The definitions found in Michael et al. (1994), Shoham (1997) and Hayes-Roth (1995) are some examples. There are a lot more definitions on agents. Each definition specifies different properties that an agent should have.

Many authors defines agents as software entities, which we think is inappropriate. Though agents are normally software entities, hardware devices and humans may also be seen as agents, if they act on someone else's behalf, with proper delegation and authorisation. Generally speaking, an agent acts on the behalf of someone else who has the right to delegate their job functions to agents, without transferring their responsibilities. We define an agent as follows, which will be used in our work:

An agent is a software entity, hardware device or human, that have been delegated job functions and limited authorities from someone else, and is within or part of an environment that senses on that environment and have the ability to react on the environment or cooperate with other agents to reach a common goal, based on its internal state.

\subsection{Problems in Agent-Mediated E-commerce}

In a collaborative system, people have responsibilities and commitments, some of which can be delegated to machine agents in many roles, which then perform actions, interacting with other agents on the user's behalf (Liu and Dix, 1997). The first problem here is that agent designers tend to develop agent applications without a clear distinction between the responsibilities and roles of agents. There is also lack of techniques to determine the responsibilities of the machine agents delegated by the human users. As a result, agent designers find it hard to specify the unique intentions and beliefs of an agent at a higher level. There are currently no formal methods that designers can use for these tasks.

Another key issue in agent-mediated e-commerce is the shared meanings of the terms and concepts used in a business domain. Agents need to share a common vocabulary of the terms for their communication to be effective. 
There is not enough attention been given on this area, though some work addresses this problems, for example, Ontolingua (Gruber, 1991). A metaphor to use is to imagine two workers trying to build a computer. They must share the same understanding of the terms such as "hard disk", "CPU", "mother board", etc. Only then will their communication gain value.

There are many other problems such as security issues, interoperability issues and language compatibility in e-commerce, though they will not be addressed in this paper.

\section{ORGANISATIONAL SEMIOTICS FOR ANALYSING AGENTS BEHAVIOUR}

\subsection{Organisational Semiotics}

The organisational semiotics approach is helpful in dealing with the problems we intend to address. It is derived from semiotics, the study of signs, human communication and meanings (Pierce 1931/35, Locke, 1960). The organisational semiotic framework (Stamper, 1998), provides guidance of information analysis at six layers: Physical, Empirics, Syntatic, Semantic, Pragmatic and Social layers. The framework offers the aspects that are required for designing agent applications in both technical and human dimensions. As Stamper (1998) puts it, "the semiotic framework serves as a basic checklist for every information system problems, for if the system do not function correctly on every level of the framework, it will fail to deliver value".

Interaction of information at a technical dimension includes the agent application being able to provide accurate and reliable physical signals at the physical layer. Then at the empirics layer, when transmitting the signals across the network, noise must not make the signals equivocal. When we have reliable signs, the receiving agent across the network can then build elaborate and formal structures at the syntactic layer.

The human and social dimensions of the framework helps agent designers to analyse business processes at a business level rather than at a technology level. This level of the semiotic framework is concerned with how signs are used for communicating meanings, intentions and also the effect through the usage of signs. It views the communication of agents in a social context. Firstly, meaningful signs at the semantics layer are created by agents based on the formal structure formed at the syntactic layer. With meaningful signs, agents can then express their intentions and beliefs at the 
pragmatic layer. It can also interpret other agents' intentions and beliefs. The resulting communication then creates a commitment, judgement, expectation, beliefs and so on at the social layer. The semiotic framework can be used as a guidebook for agent designers to broaden their understanding of the signs that will be generated at different layers which will contribute to effective communication between agents. It will also aid in requirements studies of agent applications. The semantic analysis method has the potential to be used for such requirements studies in the design of agent applications, which will be discussed in more detail in the next section.

\subsection{The Semiotic Methods}

Semantic analysis has been developed as a method for systems requirements analysis. Semantic analysis is a process of finding the meanings of the problem at a business level and expresses them in a graphical form called a semantic model. The method of semantic analysis studies the repertoires of behavior for requirements elicitation and representation (Liu, 2000).

\subsubsection{Semantic analysis to identify shared meaning}

As mentioned, agents need to create meaningful signs at the semantics layer. For other agents to be able to interpret the meaning of that sign, they must have a common understanding of that sign. For example, in human communication, a shared vocabulary is needed for that communication to gain its value. Likewise, all agents involve in a communication should have a common understanding of the terms used. This vocabulary is called an Ontology (Gruber, 1991), though we think it is more appropriate to be called conceptual models or semantic models. A semantic model is a conceptualization of a subject domain. With semantic models in place, agent designers can be informed of the vocabularies and terms that is available for communicating with other agents and the meanings that are ascribed to them.

Tools for conceptual modelling of agent systems such as the Ontolingua (Gruber, 1991) and ZEUS (Collis \& Lee 1998; Collis et al. 1998) are already being developed. However, they are simply tools for representation and do not provide a formal ground for domain clarity. They are inadequate in conceptualization of a domain at a business level. Agent designers need to be able to specify the domain from a business level rather than at the development level. The resulting semantic model from the semantic analysis enables designers to build a conceptual model of that domain where meaning of the term and the dependency between each term are formally defined. Designers can then use an implementation language such as Ontolingua to translate the conceptual model into an execution format in a seamless 
transition. Semantic analysis also helps designers to identify agents and the roles they play.

\subsubsection{Semantic analysis to identify roles of agents}

When agents function together, they negotiate with one another to determine the best course of actions in a particular context. Negotiation involves the ability to express its intentions, deduce what is being communicated and interpret the intentions of other agents at the pragmatic layer. The interpreted intention then creates a commitment or belief at the social layer. Agent communication language therefore must be flexible enough to accommodate such negotiations. However, such flexibility will make it difficult to determine the semantic meanings of a communication. To facilitate such ability, agent designers have to promote internal mental states in agents. Singh (1998) suggest that this internal state includes:

- beliefs- determines what an agent imagines its world state to be

- goals- describes what states an agent would prefer

- desires- describes the agent's preferences

- intentions- determines the goals and desires of the agents

Semantic analysis enables agent designers to specify the roles of agents. When roles are determined, it is not difficult for agent designers to analyse the internal states of an agent during norm analysis, which is another method in the semiotic approach.

\subsubsection{Norm analysis to specify intentions and beliefs of agents}

Norm analysis is used for studying an organisation from the perspective of agents' behavior which are governed by norms (Liu, 2000). When agents behave in an organised way with certain regularities, they are known as norms. Norm analysis is normally carried out based on the resulting semantic model from semantic analysis. The semantic model describes the primary area of concerns surrounding an agent application. The pattern of behavior such as the ontological relationship between agents and actions are part of the fundamental norms. Normally, norm have the following structure (Stamper, 1998):

IF condition THEN subject attitude consequent

When a condition is met, it will lead the subject (agents) of that norm to adopt a certain attitude towards some consequent (a reference to something). The attitude is reflected in a speech act or a situation which results in a disposition in one's internal state of the world. By specifying norms, agent designers can specify the intentions, beliefs, expectations etc. of agents, 
depending on the different roles that they play. For example, consider the following norms:

IF one throws an apple up, THEN one will expect that it will fall down.

IF one say "It is too expensive", THEN his intention is to hope that the price can be lowered down.

IF one makes a commitment to sell an item at a price THEN he is obliged to do so.

IF two persons are declared married, THEN they are recognised as husband and wife.

In the first example, the norm reveals that there is an expectation or belief from an agent. In the second example, an agent is revealing an intention. In the third example, an agent is obliged to do something that he had promised. In the fourth example, an agent has a status that is acknowledged by others. Stamper et al. (2000) suggests there are a number of categories of norms. Norms govern humans to behave in a certain way in perceiving, recognising and assessing things, which are perceptual, cognitive and evaluative norms. Norms are also associated with power, based on which one can make decisions or delegate duties (Krogh \& Herrestad, 1996). In an agentmediated e-commerce system, norms are important because they help agent designers to identify the attitudes that agents will adopt. These attitudes in turn reveal the beliefs, intentions and goals of an agent, which are so important to facilitate the internal states in agents. Table 1 shows the type of disposition of an agent that can be revealed simply by studying the norms and the attitude that agents will adopt:

\begin{tabular}{|c|l|}
\hline Attitudes & Disposition \\
\hline $\begin{array}{c}\text { Know, expect, imagine, think, suppose, assume, believe, } \\
\text { convinced, conclude...... }\end{array}$ & Beliefs \\
\hline $\begin{array}{c}\text { Aim to, plan to, intent to, mean to, plead, to persuade, } \\
\text { want to...... }\end{array}$ & Intentions \\
$\begin{array}{c}\text { Like, deplore, hope for, desire, dislike, hate, } \\
\text { disgusted...... }\end{array}$ & Values \\
$\begin{array}{c}\text { Be obliged to, be permitted to, be forbidden to, be } \\
\text { indebted to, be bound to, be prohibited to, entitled to...... }\end{array}$ & Acts \\
$\begin{array}{c}\text { Acknowledge, deem, recognise, admit, declare, } \\
\text { proclaim...... }\end{array}$ & Perception \\
\hline
\end{tabular}

Table 1. Dispositions that can be revealed by attitudes of agents 


\section{FOUNDATION FOR AGENCY AND COMMUNICATIONS}

\subsection{Speech Act Theory (SAT)}

Work in the area of language and communication has emphasised on the role of inference and context. Following the publication of Austin's (1962) 'How to Do Thing with Words', the speech act theory has been developed by Searle (1969). It has been further developed and used continually by linguist, philosophers, psychologist and cognitive scientist alike (Kimbrough $\&$ Moore 1997). The strength of the theory lies in its ability to classify each sentence or utterance into its corresponding act.

To illustrate the theory, consider the following example:

If John were to say:

"I promise to give you ten dollars tomorrow"

John had made a promise by the words that he used. The promise was made according to the choice of words that he used. The context which the words are used also makes a difference. Consider the following sentence:

"There's a tiger in the jungle"

- Under the context of describing the jungle, that sentence is a statement or assertion.

- However, if you tell me that you intend to take a short cut through the jungle, then under this context, that sentence is a warning.

- If you tell me that you are going hunting, that sentence then becomes a piece of advice or suggestion.

Communication languages tend to be based on speech act theory. The best known work on agent communication language is that by the APRA knowledge effort (Patil et al., 1992). This work has been devoted to developing $\mathrm{KQML}$, which will be discussed next.

\subsection{Knowledge Query and Manipulation Language (KQML)}

$\mathrm{KQML}$ is a communication language designed for software agents to exchange high level information and knowledge. It was conceived both as a message format and a message-handling protocol to support run-time knowledge sharing among agents (Finin et al., 1997). KQML is an evolving standard Agent Communication Language.

The communication function of KQML is divided into three layers: the content, message and communication layer as shown in figure 1. 


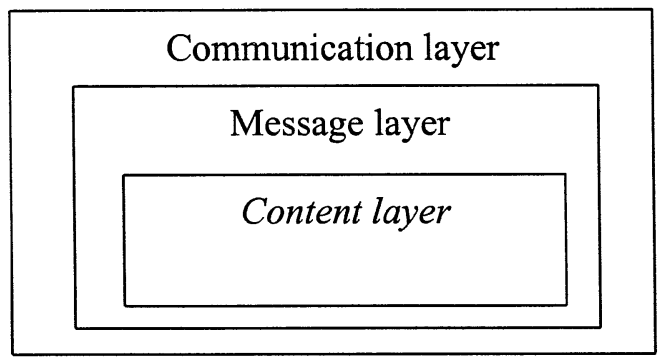

Figure 1. An abstract view of the KQML language (Nwana and Wooldridge, 1996)

\subsubsection{Communication layer}

The communication layer is based on the current network technology such as TCPIP, HTTP etc. It encodes low level parameters for communication such as specifying the sender and receiver of a message, the message ID (e.g. in-reply-to) and a unique identifier of a particular speech act. However, sometimes the communication will not involve network usage. Such communication occurs when agents reside in the same machine.

\subsubsection{Content layer}

The content layer specifies the intended content of the message expressed in a certain language and ontology. The ontology here refers to a shared vocabulary of terms. KQML is indifferent to the format of the content. In fact, the KQML standard itself has nothing to say about this. Therefore, a KQML content could be expressed in Prolog code, C code, Java code, KIF, SQL, LISP or whatsoever language, as long as it is ASCII representable.

\subsubsection{Message layer}

The message layer forms the core of KQML language. This layer of abstraction tells the receiver what actions should be executed. Most of the elements, called performatives, are used to communicate attitudes about information. There are 36 performatives and they can be classified into nine main categories which are shown in table 2 . This layer also specifies the protocol for transmitting the message and the content. By protocol, we mean the rules that must be used when agents initiate an exchange and maintaining that exchange. There are several protocols in KQML (Nwana and Wooldridge, 1996). 


\begin{tabular}{|l|l|}
\hline Category & Reserved performative names \\
\hline $\begin{array}{l}\text { Basic informational } \\
\text { performatives }\end{array}$ & tell, deny, untell, cancel \\
\hline $\begin{array}{l}\text { Basic query } \\
\text { performatives }\end{array}$ & $\begin{array}{l}\text { evaluate, reply, ask-if, ask-about, ask-one, ask- } \\
\text { all, sorry }\end{array}$ \\
\hline $\begin{array}{l}\text { Multi-response query } \\
\text { performatives }\end{array}$ & stream-about, stream-all \\
\hline $\begin{array}{l}\text { Basic effector } \\
\text { performatives }\end{array}$ & achieve, unachieve \\
\hline $\begin{array}{l}\text { Generator } \\
\text { performatives }\end{array}$ & standby, ready, next, rest, discard, generator \\
\hline $\begin{array}{l}\text { Capability definition } \\
\text { performatives }\end{array}$ & advertise \\
\hline $\begin{array}{l}\text { Notification } \\
\text { performatives }\end{array}$ & subscribe, monitor \\
\hline $\begin{array}{l}\text { Networking } \\
\text { performatives }\end{array}$ & $\begin{array}{l}\text { register, unregister, forward, broadcast, pipe, } \\
\text { break }\end{array}$ \\
\hline $\begin{array}{l}\text { Facilitation } \\
\text { performatives }\end{array}$ & $\begin{array}{l}\text { broker-one, broker-all, recommend-one, } \\
\text { recommend-all, recruit-one, recruit-all }\end{array}$ \\
\hline
\end{tabular}

Table 2. Performatives of KQML

\subsubsection{Performatives}

Besides being a message carrier, the main function of a performative is to say something about that message. It informs the receiver whether a message is a question, assertion of a fact, an answer, request, or a denial to a previous question. The protocol is also specified in the performative (e.g. what response is required? A single answer or a series of answer, an instant response to a question or a delayed answer but a guaranteed one?)

\subsection{Evaluation of KQML using Organisational Semiotics}

As KQML is still an evolving standard ACL, there will inevitably be some shortcomings in it. Organisational semiotics (Stamper, 1998) provides a powerful approach for us to evaluate KQML. In this section, KQML will be evaluated using the upper three layers of the semiotic framework and its shortcomings will be discussed. The lower three levels will not be considered as they are more concerned with the physical properties of signs, how signs are organised and transmitted and how signs are structured and used in language. The focus of this evaluation is on the use of KQML to communicate meanings, to communicate intentions and the social effects through the use of KQML. 
Firstly, let us consider the semantic level of the semiotic framework. For a successful communication to take place, the people involved in that communication must have a common semantic meaning of the terms that are used. KQML, like all programming languages, does not have a formal and precise semantic meaning of the performatives. The only way to describe the intended semantic meanings of the performatives and their use are currently restricted to natural language descriptions (Finin, 1997). As a result, agent designers do not have a common understanding of the language, its performatives and protocol. Cohen and Levesque (1995) expressed this concern by stating that agent designers cannot be certain whether the same interpretation that they give to a performative can be unambiguously understood by other designers.

Secondly, at the pragmatic level, intentions, beliefs, etc. can be communicated with the existence of a shared body of knowledge or meanings at the semantic level. Since no one is clear how to give the performatives a precise definition at the semantic level, intention and beliefs cannot be communicated unambiguously at the pragmatic level. For example, the interpretation that a designer gives to the ask-one performative may be different from the interpretation of another designer, which will then cause confusion. It is therefore not possible to determine whether any agent application is KQML compliant. As Singh (1998) puts it, "if you cannot determine compliance, that standard is useless".

When intentions can be unambiguously communicated at the pragmatic level, it creates a commitment or belief at the Social level. However, due to a lack of performatives in KQML, designers cannot create the kind of beliefs that he intend the receiving agent to have. The following section in this paper also argues that KQML misses a few important classes of performatives.

Singh (1998) states that there are seven main categories of communicative acts:

Assertives- the speaker informs or states a fact and he is committed to the truth of that fact. Example: "It is hot"

Directives- the speaker attempts to get the recipient to do something for instance request, ask, and command. Example: "Open the door".

Commissives- the speaker is committed to a certain course of actions that was uttered. Example: "I promise to go home before 5.00pm"

Permissives- the speaker gives permission for the recipient to do an act. Example: "You may go".

Prohibitives- the speaker bans the recipient to do an act. Example: "You may not leave the office before $5.00 \mathrm{pm}$ " 
Declaratives- the speaker attempts to alter the external status of an object or circumstance and cause event solely by uttering the act. Example: "I declare you husband and wife".

Expressives- the speaker expresses emotions and physchological attitude about a state of affairs. Example: "Thank you for coming".

First of all, before evaluating whether the performatives in KQML are adequate for use in an agent-mediated e-commerce system, let us consider the basic phases that will be involved in a simple deal in e-commerce. There are four basic phases (see figure 2), which form a co-ordinated process between two agents, the buyer and the supplier. In the first phase, the buyer requests for some products or services from the supplier. In the second phase, the buyer and supplier negotiate on the terms of the deal such as delivery dates, prices and so on. In the third phase, the supplier commits on the deal, for instance the prices and performs the work. In the fourth phase, the supplier has to provide support of their products to the buyer. These four phases give a very general description of a business deal. One does not have to take all the four phases to complete a deal. In an actual business deal, there may be more phases like raising a purchasing order, sending an invoice and so on. But these are the four main ones. Table 3 shows the associated speech acts in these phases.

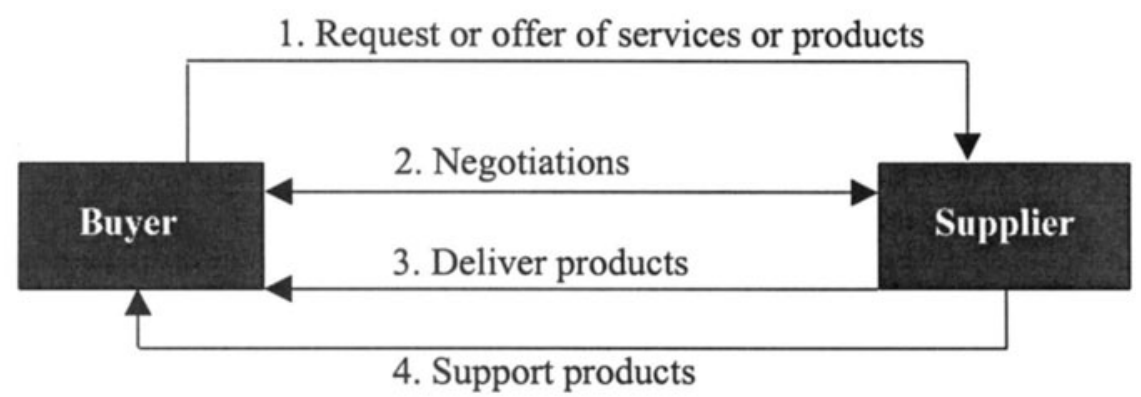

Figure 2. The main phases of a deal in electronic commerce

To illustrate the inadequacy of the performatives in KQML, we want to particularly comment on phase two. At the end of a negotiation process, the seller may promise to sell the products at a price that the buyer agree upon. Likewise, the buyer may also promise to buy from the seller if that price remains unchanged. KQML needs to support this characteristic of making commitments and promise but the performatives in KQML have limited coverage. All performatives, as Singh (1998) argued, are either assertives or directives. With only a limited and small set of performatives, there is a possibility that overloading may occur at the content lever. 


\begin{tabular}{|l|l|l|}
\hline Phases & Agent & Speech acts \\
\hline Phase 1: Request & $\begin{array}{l}\text { Buyer } \\
\text { Seller }\end{array}$ & $\begin{array}{l}\text { Request, Ask } \\
\text { Offer }\end{array}$ \\
\hline Phase 2: Negotiation & Buyer & $\begin{array}{l}\text { Request, Insist, Suggest } \\
\text { Promise/Commit, Refuse }\end{array}$ \\
\cline { 2 - 3 } & Seller & $\begin{array}{l}\text { Offer, Insist, Suggest, } \\
\text { Promise/Commit, Refuse }\end{array}$ \\
\hline Phase 3: Delivery & Buyer & Acknowledge \\
\cline { 2 - 3 } & Seller & Thank \\
\hline Phase 4: Support & Buyer & Satisfied, Unsatisfied, Request \\
& Seller & $\begin{array}{l}\text { Apologise } \\
\text { Offer }\end{array}$ \\
\cline { 2 - 3 } & &
\end{tabular}

Table 3. Four phases of a deal and their corresponding speech acts

While we do not deny that providing an overly extensive set of performatives will results in narrowing down the distinction between different performatives, we argue that performatives like commisives, prohibitives etc. should be included for agents to be able to engage in a more complex social relationship especially in e-commerce.

\section{SEMIOTIC METHODS FOR DESIGNING E- COMMERCE SYSTEMS}

To illustrate how semiotic methods can help in designing an e-commerce system, the case of an online bookstore will be used. The analysis begins with a problem description as follows:

"A bookstore, employing a number of staff, offers many books to be sold online. The online bookstore has a Web address where the online catalogue is stored. Customers have the ability to search for books, using the online catalogue. The search can be done in various options such as book title, $I S B N$, author. Customers can then know the price of the books. If he wishes to buy that book, he submits his credit card number and the details of the book. This transaction then initiates a transfer of funds from the customer's credit card company to the bookstore. The bookstore is committed to deliver the book within three days of ordering. Prices indicated are inclusive of delivery charges." 


\subsection{Architecture of Agent-Mediated E-Commerce System}

The proposed architecture of the e-commerce system is shown in figure 3 , on which the rest of the discussion will be based. In figure 3 , we show the relationship between conceptual model, agents and the dynamic aspect of agent behaviour (norms). All agents will refer to the conceptual model and be subjeted to the norms for their behaviour.
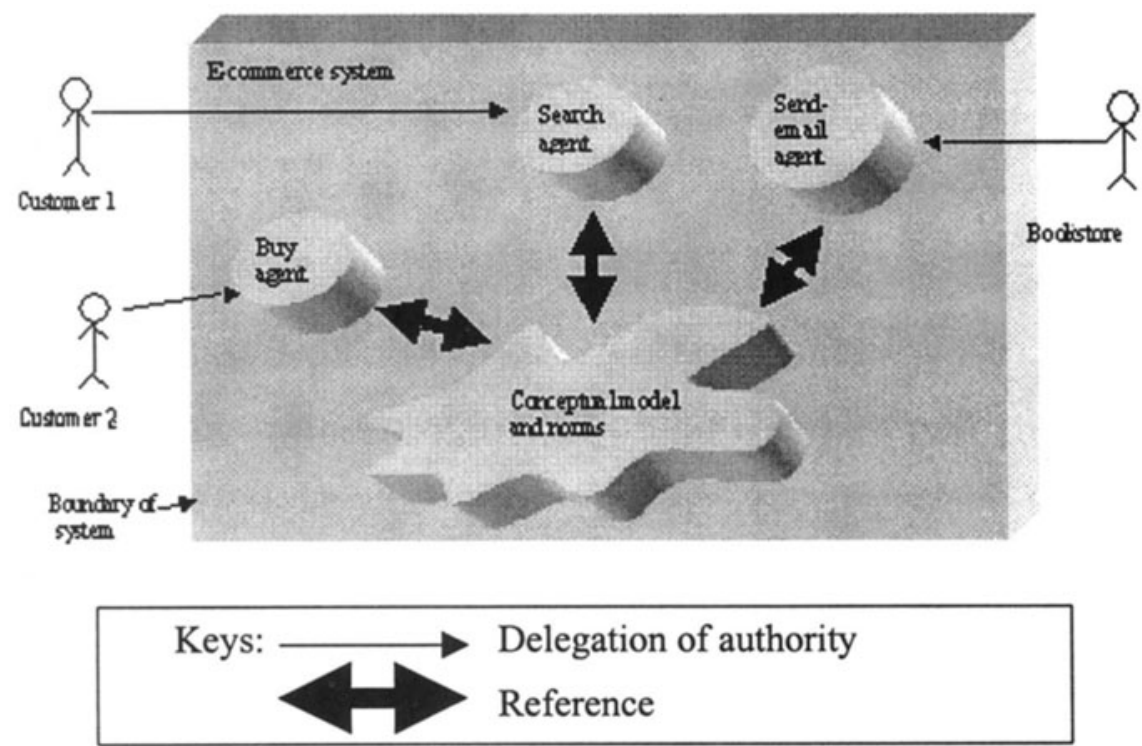

Figure 3. Proposed architecture of agent-mediated e-commerce system

\subsection{Conceptual Model Shared By Agents}

The method of semantic analysis is applied at this stage to obtain a conceptual model that is shared by all the agents. Semantic units are identified from the problem definition and grouped. Words or phrases are singled out as candidate semantic units. Every word in the problem definition is important as they may carry some information to a concept, a relationship or an intention. As a result, the following lists are some examples of the candidate entities that are obtained:

Bookstore, Employee, Shipped, Address, Books, Buy, Sell, etc.

After the functions of candidate entities are listed and studied, they are classified into a list of entities, patterns of behaviour, role-names and attributes. Some examples are shown in table 4. At this stage, we may have developed sketches of piecemeal semantic structure as shown in figure 4 . 


\begin{tabular}{|ll|}
\hline Bookstore: & Legal entity of business which owns and sells books \\
\hline Employer: & A role name of an agent who employs staffs \\
\hline Invoice: & An patterns of behaviour of Bookstore \\
\hline Price & An attribute of books \\
\hline
\end{tabular}

Table 4. Some semantic candidates

Some candidate entities did not appear literally in the list, for example, "seller" and "buyer" are incorporated into the representation implicitly by introducing a pattern of behaviour called "trade". Some new terms such as society, person, buyer and seller are introduced in the grouping in order to construct a sound semantic structure.
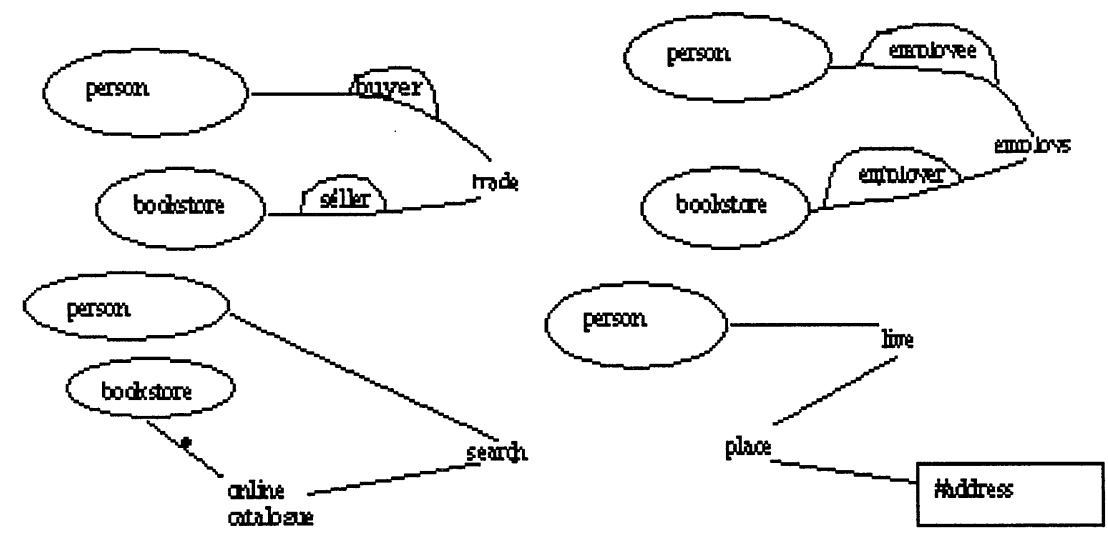

Figure 4. Piecemeal of semantic structure

The fragments in figure 4 will be assembled into an integrated semantic model as shown in figure 5. The semantic model is a conceptual model in a given domain. Entities and patterns of behaviour are nodes in this model and society is the root agent which defines the boundary of the study. The entities are normally human actors or organisations that have authority and can delegate responsibilities to machine agents. As seen in figure 5, the Society has three patterns of behaviour, books, person and bookstore and all three are ontologically dependent on the Society. That means if Society does not exist, bookstore, person and book will also become undefined.

The model shows clearly the roles of person. Role names are in semicircles. For example, in this domain, the entity person can have the role of a buyer or an employee of the bookstore. When we can identify the roles that each agent have to perform, it is not difficult to also specify the beliefs and intentions in the next phase which is known as the norm analysis. 


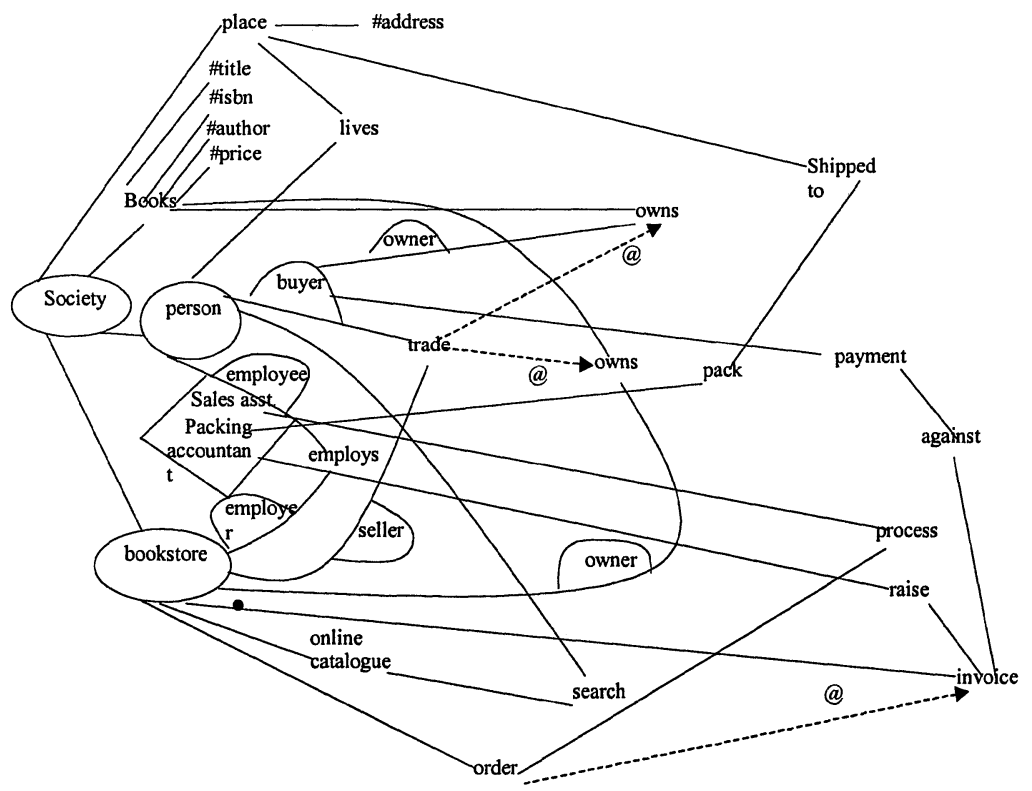

Figure 5. Semantic model of online bookstore

Using the semantic model that we already have (see figure 5), we attempt to find out the disposition of the agents based on their different roles.

\subsection{Capturing the Dynamics of Agent Behaviour in Norms}

In this section, we capture the norms and derive the attitude and disposition of agents. The attitudes are reflected in a speech act or a situation which results in a personality, called disposition, in one's internal state of the world. Table 5 shows some examples of the norms governing the behaviour of agents in the bookstore case study.

The norms specify conditions and constraints for certain events and actions. Agents are normally involved in these events and actions with attitudes and dispositions in their internal state of the world. Once these norms are elicited, they can then be specified further for execution.

\subsection{Implementation of E-Commerce Systems}

As displayed in figure 3, the semantic model and norms will be the conceptual model for designing the e-commerce system for the bookstore. All agents that serve the bookstore will share the same semantic model and the same set of norms. 


\begin{tabular}{|l|l|l|l|}
\hline Agent & Norms & Attitude & Disposition \\
\hline Buyer & $\begin{array}{l}\text { IF buyer wish to buy a book, } \\
\text { THEN he will plan to search } \\
\text { for that book }\end{array}$ & Plan & Intentions \\
\hline Buyer & $\begin{array}{l}\text { IF buyer has ordered a book, } \\
\text { THEN he is obliged to make } \\
\text { payment }\end{array}$ & Obliged & Acts \\
\hline Owner & $\begin{array}{l}\text { IF bookstore owns a book, } \\
\text { THEN he is permitted to sell } \\
\text { it }\end{array}$ & $\begin{array}{l}\text { Permitted to } \\
\text { the boller has promised to sell } \\
\text { is obliged to sell at that price }\end{array}$ & Octs \\
\hline Buyer/seller & $\begin{array}{l}\text { IF a trade is successful, } \\
\text { THEN the transfer of } \\
\text { ownership of books between } \\
\text { seller and person can be } \\
\text { acknowledged }\end{array}$ & $\begin{array}{l}\text { Acknowled } \\
\text { ged }\end{array}$ & Perception \\
\hline
\end{tabular}

Table 5. Disposition revealed by norms

These provide the unifying basis for agents to communicate at the semantic and pragmatic levels with shared meanings and concepts. To validate this hypothesis, an experiment is being carried out by prototyping the agent-mediated system using an agent-building CASE tool ZEUS (Collis et al. 1998).

\section{DISCUSSION AND FURTHER WORK}

Firstly, we showed how agents are involved in agent mediated ecommerce applications. The definition of agents and their properties were discussed. Secondly, we showed the problems of agent-mediated ecommerce. The semiotic framework was introduced and was used to evaluate KQML. Also, the shortcomings and inadequacy of KQML was discussed.

Thirdly, semiotic methods were presented for designing agent-mediated e-commerce systems. Using a case study of a bookstore, we showed how semantic analysis enables system developers to identify agents and specify its roles. The norm analysis was use to identify the norms, the agents involved, the attitude that they will adopt and eventually, the disposition of 
an agent so that the internal states of agents can be properly and accurately coded.

Many opportunities for future work exist. This may lead to an extension of KQML so that it can be used by agent mediated e-commerce systems to specify a more complex relationship. Applying semiotic methods into analysis and modelling agent behaviour within the constraints of responsibilities and authorities set by human users in e-commerce is an important follow-up that will be carried out in the near future. Another task is to develop a formal syntax and semantics for specifying norms and disposition to facilitate analysis and design of internal states (beliefs, intentions) of agents.

\section{References}

Adams, E.J. (1994) "Electronic Commerce Focusing On Success", Chain Store Age Executive.

Austin, J.L. (1962) "How to Do Things with Words", Oxford at the Clarendon Press, Oxford, U.K.

Boynton, A.G. (1994) "Electronic Commerce Focusing on Success", Chain Store Age Executive.

Cohen, P.R. and Levesque, H.J. (1990a) "Intention Is Choice With Commitment", Artificial Intelligence, 42, pp. 213-261

Cohen, P.R. and Levesque, H.J. (1990b) "Rational Interaction As The Basis For Communication". In Cohen, P.R., Morgan, J., Pollack, M.E. (Eds.), Intentions in Communication, The MIT press: Cambridge, MA, pp. 221-256

Cohen, P.R. \& Levesque H.J. (1995) "Communicative Actions for Artificial Agents", In Proceeedings of the First International Conference on Multi-Agent Systems, San Francisco, Cambridge: AAAI Press pp. 65-72

Collis, J.C. \& Lee, L.C. (1998) "Building Electronic Marketplaces with the ZEUS Agent Toolkit", Proceedings of the Agent Mediated Electronic Trading (AMET) Workshop, May, pp. 17-32

Collis, J.C., Ndumu, D.T., Nwana, H.S. \& Lee, L.C. (1998) “The ZEUS Agent Building Toolkit" BT Technology Journal, 16(3), pp. 60-68

Finin, T., Fritzson, R., McKay, D. \& McEntire, R. (1994) "KQML as an agent communication language", In Proceedings of $3^{\text {rd }}$ International Conference on Information and Knowledge Management (CIKM), November, New York: ACM Press.

Finin, T. Labrou, Y. Mayfield, J. (1997) "KQML as an agent communication language". In J. Bradshaw (Ed.) Software Agents, MIT Press, Cambridge.

Fisher, J. (1995) "Electronic Commerce, A Definition", AIIA/ECA Publication on Electronic Commerce, February

Gruber, T. (1991) "Ontolingua: A Mechanism to Support Portable Ontologies", KSL-91-66, Stanford University Knowledge Systems Laboratory

Hayes-Roth, B. (1995) “An architecture for adaptive Intelligent Systems" Artificial Intelligence: Special Issue on Agents and Interactivity, Vol. 72 
Hornback, R. (1995) "Electronic Commerce in the $21^{\text {st }}$ Century", Journal of Systems Management, May/June, pp. 28-33

Kimbrough \& Moore (1997) "On Automated Message Processing in Electronic Commerce and Work Support Systems: Speech Act Theory and Expressive Felicity", ACM Transactions on Information Systems, Vol. 15(4), pp. 321-367

Krogh, C. \& Herrestad (1996) "Getting Personal: Some Notes on the Relationship Between Personal and Impersonal Obligation", in Brown, M.A. \& Carmo, J. (Eds.) Deontic Logic, Agency and Normative Systems, Springer

Lederer, A.L., Mirchandani, D.A., Sims, K. (1996) "Electronic Commerce: A Strategic Application”, SIGCPR/SIGMIS, Denver Colorado USA, pp. 277-287.

Locke, John (1960) "Essay Concerning Human Understanding”, Unabridged edition 1959, Dover, NY

Liu, K., Apers, P., Chattopadhyay, S., Cheng, A., Fox, M., Greene, T., Klein M. \& Stamper, R. (1999) "Enterprise Information Systems: issues, challenges and viewpoints". In J. Filip (Ed.), Enterprise Information Systems, Kluwer Academic Publishers (in press).

Liu, K. (2000) "Semiotics in Information Systems Development" Cambridge University Press (in press)

Liu, K. and Dix, A.(1997) "Norm Governed Agents in CSCW", 1st International Workshop on Computational Semiotics, University of De Vinchi, Paris.

Mowshowitz (1997) "Virtual Organisation", Communications of the ACM 40(9), pp. 30-37

Michael R., Genesereth, Steven P. K. (1994) "Software Agents", Communications of the ACM 37(7) pp. 48-53

Singh, M P. (1998) 'Agent Communication Language: Rethinking the Principles', Computer: Innovative Technology for Computer Professionals, December, pp. 40-47

Nwana H. S., Michael Wooldridge (1996), "Software Agent Technologies", BT Technology Journal. Also available from: http://www.labs.bt.com/projects/agents/publish/papers/sat_report.html

Collis, J.C., Ndumu, D.T., Nwana, H.S. \& Lee, L.C. (1998) “The ZEUS Agent Building Toolkit" BT Technology Journal, 16(3), pp. 60-68

Patil, R.S., Fikes, R.E., Patel-Schneider, P.F, McKay, D, Finin, T., Gruber, T., and Neches, R. (1992) "The DAPRA Knowledge Sharing Effort: Progress Report." In Rich, C., Swartout, W. and Nebel, B., (Eds), Proceedings of Knowledge Representation and Reasoning, pp 777-788

Pierce, C.S. (1931/35) "Collected Papers of C.S., Pierce (1931-35)", edited in 1960 by Hartshorne, C. \& Weiss, P., Cambridge, Mass

Searle, J.R. (1969) "Speech Acts", Cambridge University Press, Cambridge, U.K.

Shoham, Y. (1993) “Agent Oriented Programming” Artificial Intelligence, Vol. 60 pp.51-92

Shoham, Y. (1997) "An Overview of Agent-Oriented Programming”, Software Agents, Bradshaw, J.M.(ED), AAAI Press, Menlo Park, CA, USA

Spence, M.A. (1994) 'Electronic Commerce Is The First Beneficiary of The Information Highway', CAM Magazine, June, pp. 6

Stamper (1998) “Organisational Semiotics". In John Mingers \& Frank Stowell (Eds.), Information Systems: An Emerging Discipline?, The McGraw Hill Companies, pp.267-283 